\title{
Provider-initiated HIV counselling and testing (PICT) in the mentally ill
}

\author{
M Y H Moosa, FC Psych, MMed Psych, MCFP, MB ChB; F Y Jeenah, FC Psych, MMed Psych, MB ChB \\ Division of Psychiatry, Faculty of Health Sciences, University of the Witwatersrand, Johannesburg, South Africa
}

Corresponding author: M Y H Moosa (yusuf.moosa@wits.ac.za)

\begin{abstract}
The prevalence of HIV infection is substantially higher in mentally ill individuals than in the general population. Despite this, HIV testing is not yet standard practice among the mentally ill population, and many mental health settings do not encourage HIV testing. This paper discusses provider-initiated HIV counselling and testing (PICT) and some of the ethical dilemmas associated with it, on the basis that PICT may be used to increase the number of mentally ill persons tested for HIV. The authors conclude that PICT should be promoted to all psychiatric admissions and mentally ill individuals receiving outpatient services, and that this is within the parameters of existing policies and legislations in South Africa.
\end{abstract}

S Afr J Psych 2013;19(3):60-64. DOI:10.7196/SAJP.408

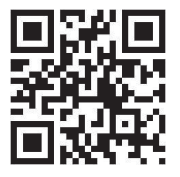

The Joint United Nations Programme on HIV and AIDS (UNAIDS) reported that the global number of people newly infected with HIV was declining and that AIDS-related deaths were decreasing in $2010 .{ }^{[1]}$ In the same report UNAIDS estimated an HIV prevalence rate of $17.8 \%$ in South Africa (SA), based on studies including the Antenatal ${ }^{[2]}$ and Household ${ }^{[3]}$ Surveys which reported rates of $29 \%$ and $11 \%$, respectively. Although the prevalence rate in SA appeared to have stabilised, it was still high compared with the rest of the world. ${ }^{[1]}$

Routine HIV testing among the mentally ill in SA is not yet standard practice, despite the high prevalence of HIV, the strong association between the virus and mental illness, and the risk of acquiring and transmitting HIV. We propose that provider-initiated HIV counselling and testing (PICT) could be a tool for increasing the number of mentally ill persons tested for HIV. However, there are a number of ethical dilemmas associated with this form of testing, which this paper addresses.

\section{Increasing HIV testing rates in the mentally ill through the PICT approach}

The World Health Organization (WHO) reports that worldwide in 2005 , a mere $8-20 \%$ of people living with HIV knew their serostatus. ${ }^{[4]}$ According to the WHO, the number of people being tested for HIV worldwide increased to $25 \%$ of the adult population in 2009 and to $35 \%$ in $2010,{ }^{[5]}$ and a similar trend was seen in SA. The 2010 National Communications Survey included 9728 people aged 16 - 55 years, from all 9 provinces and who were representative of the country's population. The survey reported that approximately $60 \%$ of men and women had been tested in the 12 months prior to the study, an increase of about $36 \%$ since the 2009 survey. ${ }^{[6]}$

Although the availability of HIV testing in public health facilities has increased in recent years, uptake of HIV testing is still relatively low. ${ }^{[7]}$ Increasing these rates was the main objective of the South
African National HIV Counselling and Testing (HCT) Programme, ${ }^{[7]}$ which takes two approaches to testing: (i) client-initiated counselling and testing (CICT); and (ii) PICT. In CICT, also referred to as voluntary counselling and testing (VCT), individuals actively seek HIV testing and counselling at a facility that offers these services, as well as pre-test information. The process is voluntary and the 'three Cs' (informed consent, counselling and confidentiality) are observed at all times. ${ }^{[7]}$ In PICT (also referred to as the routine offer of counselling and testing, or 'opt-out testing') testing is initiated by healthcare providers and recommended to all clients attending healthcare facilities, as a standard component of medical care. ${ }^{[4]}$

PICT aims at early identification of clients for whom there may be a strong likelihood of HIV infection, because of their symptoms, their high-risk sexual behaviour or their location in areas of high HIV prevalence. The 'opt-out approach' means that HIV testing is done together with all other relevant tests, unless the client actively or explicitly refuses HIV testing. Such refusal is documented in the client's clinic file. In contrast, in the 'opt-in approach' the client must specifically agree to the test rather than having to refuse it. With both approaches, the service provider recommends the testing as a standard part of medical care, and the purpose of testing is to enable specific clinical decisions to be made and/or specific medical services to be offered that would not be possible without knowledge of the person's HIV status. ${ }^{[8]}$ The PICT approach protects and promotes the client's right to autonomy and dignity, while recognising the duty of healthcare providers to protect the right to life and access to health services. ${ }^{[7]}$ This HCT Programme has resulted in an increase in HIV testing in the general population.

However, the prevalence of HIV infection in mentally ill individuals is substantially higher than in the general population. In the USA, prevalence rates in mentally ill patients are reported at $5-23 \%$, compared with $0.3-0.4 \%$ in the general population. ${ }^{[9]}$ Similar trends have been reported in African studies. ${ }^{[10-12]}$ The higher prevalence rate 
may be attributed to reportedly higher rates of sexual risk behaviour (multiple partners, not using condoms, and history of STDs) among adults with mental illness compared with the general population. ${ }^{[13]}$ The reasons for risky behaviour include:

- psychiatric symptoms such as hypersexuality and erotomania

- consequences of mental illness, such as lack of planning ability, poor risk assessment, and poor communication skills

- insufficient information, motivation and skill to engage in safe sexual behaviours

- the social drift associated with mental illness (e.g. poverty, homelessness), resulting in no money for condoms and no privacy for safer sex negotiation

- hospitalisation, which impedes long-term relationships and the formation of social support structures

- substance use.

Mental health problems are associated with an increased risk of acquiring and transmitting HIV and may also interfere with the treatment of HIV. Individuals with mental illness should be tested for the benefit of both individual and public health: ${ }^{[14]}$ those who test positive will benefit from receiving medical care, while antiretroviral therapy will reduce infectiousness and knowledge of one's HIV status motivates risk reduction, which is of public benefit. ${ }^{[15]}$

Despite the strong association between mental illness and HIV, routine HIV testing is not yet a standard practice among the mentally ill population and many mental health settings do not encourage HIV testing. Studies indicate that the percentage of this population group that has been tested is very low, between $17 \%$ and $47 \% \cdot{ }^{[13,16]}$ In a survey of psychiatric hospitals and outpatient mental health centres, the majority of staff reported that they only encouraged a few patients to get tested. ${ }^{[17]}$ Joska et al. ${ }^{[18]}$ report that state psychiatrists in SA's Western Cape Province do not test routinely for HIV infection (14.6\% of patients with serious mental illness were tested in 2006), largely because of ethical constraints.

Studies investigating correlates of low testing among individuals with mental illness have reported no clear association between HIV testing and either demographic or psychiatric variables. ${ }^{[19,20]}$ However, low testing rates have been linked to a reliance on VCT as the sole approach to HIV testing. ${ }^{[21]} \mathrm{VCT}$ is predominantly provided by lay counsellors who are insufficiently trained in mental illness, which makes them uncomfortable about delivering risk reduction counselling and obtaining consent from the mentally ill. ${ }^{[22]}$ On the other hand, mental health professionals can routinely offer PICT to all patients as a standard part of psychiatric care, regardless of patients' presenting complaint. PICT would still be voluntary as the patient is given the option to 'opt-out' of testing. ${ }^{[8]}$ Ideally, mental health clinicians can provide pre- and post-test counselling that is tailored for individuals with a mental illness, addressing patients' barriers to testing and allaying concerns about testing, confidentiality and stigma. ${ }^{[17]}$ Mental health settings are also ideal because patients located here avoid the need for additional transportation or appointments. ${ }^{[17]}$ HIV testing could be recommended during consultation, together with all other relevant tests. ${ }^{[23]}$ If the patient actively refuses HIV testing, this would be documented in their clinic file. Ultimately, patients who are tested will then be given the test result and provided with post-test counselling by mental health professionals.

\section{Addressing the ethical challenges}

CICT is a voluntary process wherein patient autonomy is ensured by observing the 'three Cs' (informed consent, counselling and confidentiality). ${ }^{[7]} \mathrm{PICT}$ is also considered to be ethically legitimate, especially when the three Cs are actively practised. ${ }^{[24]}$ The WHO/ UNAIDS guidance recommends the opt-out approach, as it depends on the patient's informed decision to accept or decline the healthcare provider's recommendation of an HIV test. ${ }^{[8]}$ PICT is an attempt to balance autonomy with other ethical demands, such as equality and beneficence, which are relevant in an already marginalised mentally ill population. When implementing the PICT approach, however, clinicians may still face ethical and legal challenges such as ensuring that the consent obtained is indeed informed and that individuals who test positive have access to appropriate care. ${ }^{[25]}$

\section{Consent}

Informed consent is often described as comprising disclosure of information, comprehension of that information and a voluntary choice. HIV testing must always be voluntary and free of coercion. In order for any person to make an informed decision and to give consent, they should be given information about HIV acquisition and transmission, HIV risks and risk reduction, the importance of early HIV diagnosis, the testing process, the meaning and implications of a negative result and the window period, and finally the meaning and implications of a positive result and disclosure.

When obtaining consent from a mentally ill person, the issue is whether they have the cognitive capacity to comprehend all this information and make an informed decision. Mental health professionals are in the best position to determine this through a clinical assessment of the mental status necessary to give consent, rather than by making inferences from a specific diagnosis of mental illness. ${ }^{[26]}$ Most patients with mental illnesses are stable and do not have impairments that affect their ability to give consent. Even patients with serious mental illnesses (SMI), such as schizophrenia, bipolar disorder and recurrent major depression, which can produce cognitive impairment, understand their illness and the need for medical interventions (including laboratory testing) and can give informed consent for said interventions. ${ }^{[26]}$ It may be concluded that the majority of mentally ill patients, despite some compromise of their cognitive abilities, are in a position to give voluntary informed consent for testing.

One could argue that testing for HIV may not have any relevance to the patient in their current situation, and that therefore such a medical intervention is not warranted. The same could be said for other tests to exclude medical illness. However, the discussion here is not about the relevance of the tests at the time but rather the capacity to make an informed decision and the maintenance of the individual's autonomy. Therefore, in such cases, the mental health professional can recommend PICT knowing that if the patient does not actively refuse, then the tacit consent is valid and the patient's autonomy is not compromised. If there are any doubts about the patient's capacity to consent, the clinician could be assisted with some objective research instruments such as the MacArthur Competency Assessment Tool for Clinical Research ${ }^{[27]}$ and the University of California, San Diego Brief Assessment of Capacity to Consent (UBACC). The UBACC, a 10 -item scale that can be administered by bachelor's degree-level staff, 
typically takes less than 5 minutes and provides for the assessment and documentation of participants' understanding and appreciation of elements essential to meaningful consent and the voluntary nature of participation. ${ }^{[28]}$

However, some acutely ill patients with SMI cannot give informed consent because they do not understand the nature and purpose of a medical intervention, are unable to choose decisively, or are unable to communicate their consent. Other mental illnesses that affect cognition and a person's ability to give informed consent include delirium, dementia, and intellectual disability. The laws requiring informed consent for HIV testing in these individuals differ in different countries, and the need for testing without consent is decided on an individual basis. The South African National Health Act $^{[29]}$ states that if an adult client is unable to give informed consent, such consent can be given by a person authorised in terms of any law or court order, i.e. the spouse or next-of-kin (parent, grandparent, an adult child or a sibling, in the order listed). Similarly, the Mental Health Care $\mathrm{Act}^{[30]}$ requires that if a mentally ill person is unable to consent or cannot be restored to a state where he/she is able to consent, a curator, spouse or family member may provide consent.

In the absence of the above and in cases of emergency, consent may be obtained from the head of the health establishment (HHE). In such cases of SMI, PICT can be recommended and consent obtained from a legally authorised person. This approach also complies with the ethical principles of beneficence and the duty of care, which justifiably overrides the autonomy of the patient with impaired cognitive function when there is a risk to the individual and/or society at large. Individuals who test positive may benefit by receiving medical care and antiretroviral therapy (ART) to reduce infectiousness and restore good health. ${ }^{[15]}$ If they do not meet the criteria for ART, disease progression can be monitored over the long term. Equally importantly, patients who know their HIV status may enter programmes on the reduction of risky sexual behaviour, which is of public benefit. ${ }^{[15]}$

However, some clinicians argue that the legal procedures of obtaining consent from the family or the HHE can sometimes take days to weeks, which may lead to deterioration in the individual's mental and physical health. Joska et al. ${ }^{[18]}$ recommend that current legislation be amended to allow the psychiatrist in charge of the patient to provide consent. This would allow for the immediate delivery of the most appropriate care. Further, families and the HHE are often not psychiatrically trained and may not fully understand the complexities and the need to do an HIV test without consent. This approach will also maintain patient confidentiality and may avert the risk of stigmatisation and marginalisation by the family.

\section{Treating HIV-positive mentally ill persons}

Having recommended and initiated HIV testing in mentally ill patients, the clinician is then faced with the problem of treating HIV-positive patients. Although antiretroviral treatment sites are being extensively rolled out in SA, they are not easily accessible to patients with mental illness. Some doctors delay the prescription of antiretroviral medication for individuals with an SMI due to concerns about (i) interactions among medications; or (ii) HIV-medication adherence. ${ }^{[31,32]}$
Mentally ill patients, like other patients with chronic medical conditions taking many medications, are at high risk for drug interactions. The most common drug-drug interactions with antiretrovirals and psychotropics are based on cytochrome P450 (CYP) metabolism, which is common to many psychotropics, the protease inhibitors (PIs), and the non-nucleoside reverse-transcriptase inhibitors (NNRTIs). All PIs and NNRTIs are metabolised by the CYP system and possess enzyme-inhibiting or enzyme-inducing properties. ${ }^{[33]}$ The nucleoside reverse-transcriptase inhibitors (NRTIs), as well as the newer fusion inhibitor (enfuvirtide) and integrase inhibitor (raltegravir) are not metabolised significantly by the CYP system, making them less vulnerable to interactions with psychotropic medications. ${ }^{[28]}$

Some of these newer drugs are only available in the private sector, and in the foreseeable future the majority of the uninsured population will only receive the PIs and the NNRTIs. The clinical relevance of these potential CYP interactions is variable, as it is not clear how often psychotropics adversely impact ART blood levels or what effect ART has on psychotropic blood levels (causing either toxicity or failure).

In managing drug interactions, the newer ART and psychotropic agents have a lower potential for drug-drug interactions and are therefore preferred. The use of low initial doses and slow titration of psychotropics, in combination with older ARTs, will help to minimise adverse effects. Although there still remains a potential for adverse events when combining psychotropics and antiretrovirals, the likelihood of an improved quality of life and better adherence through improved mental wellbeing warrants the risk. ${ }^{[34]}$ It is also just and ethically acceptable, as patients with other chronic medical illnesses and on drugs that potentially interact with antiretrovirals, are not prevented from receiving antiretrovirals. Safe and effective treatment of mentally ill patients with HIV infection requires the educated use of psychotropics and antiretrovirals.

The feasibility and the sustainability of antiretroviral programmes in mentally ill people has been questioned because of concerns about low and suboptimal adherence. ${ }^{[35]}$ They argue that PICT may increase the number of people being tested, but if they test positive they cannot be initiated on treatment because they will not be fully adherent to the antiretrovirals. This will lead to the development of resistant strains of HIV that may be transmitted to uninfected patients, leaving them without effective options for treatment; ${ }^{[36]}$ or require a change to second-line treatment regimens which are 10 times more expensive than first-line drugs. ${ }^{[37]}$ Although high levels of adherence (90-100\%) are theoretically needed to ensure that viral load is kept at undetectable levels, ${ }^{[38]}$ the actual reported rates of adherence in the general population worldwide vary from $50 \%$ to $80 \% .{ }^{[39,40]}$

The antiretroviral adherence rates in mentally ill patients are reported to be only slightly but not significantly lower. ${ }^{[41-43]}$ Even patients with SMI are able to adhere very well to antiretroviral regimens. ${ }^{[44]}$ Many variables other than mental illness are consistently associated with poor access and adherence to HAART and include substance abuse, HIV symptoms (such as weight loss and fatigue), medication side-effects, complexity of the treatment regimen, health beliefs, social support, cognitive functioning and self-efficacy. ${ }^{[32,45]}$ Uldall et al. ${ }^{[33]}$ concluded that there are still doubts about whether or not there is a particular mental disorder, a combination of disorders or other related factors that lead to non-adherence to HAART. 
Chander et al. ${ }^{[45]}$ reported that most of the studies of the impact of psychiatric illness on HAART adherence have largely been centred on depression and anxiety disorders, with very few randomised controlled trials. They concluded that the studies appear to contradict each other regarding the relative impact on HAART non-adherence attributable to common psychiatric disorders, and that the interaction between psychological distress, cognitive distortions, self-efficacy and physical symptoms of mental illness affecting access and adherence to antiretrovirals remains unclear. There is a need for additional research into the mechanism by which mental illnesses influence medication adherence in the HIV/AIDS population.

Mental illnesses also possess unique characteristics that require illness-specific interventions, beyond proper treatment with medications. Special attention to issues commonly associated with mental illness such as stigma, homelessness and poverty also need to be addressed. ${ }^{[46]}$ One strategy for increasing access and improving adherence to HAART among this complex population may include monitoring providers closely, and locating primary-care services in traditional settings for mental health treatment, where the mentally ill population receives the bulk of their care. ${ }^{[4]}$ Other strategies include educating and motivating patients, simplifying treatment regimens and tailoring them to individual lifestyles, preparing for and managing side-effects, and addressing the concrete issues that may present barriers to adherence. ${ }^{[48]}$ In addition, adherence-boosting interventions that have established efficacy are recommended, such as motivational interviewing and nurse-based interventions for patient populations with low health literacy. Further, support group interventions combining providers, patients and the community have been shown to improve adherence in mentally ill patients with HIV and AIDS receiving ART, focusing on the aspects of education, emotional support, stigma and skill building. ${ }^{[49]}$

In individuals with both mental illness and substance abuse or dependence, it appears that adherence is most influenced by active alcohol or drug use. These chemical dependency issues need to be addressed before people living with HIV/AIDS can successfully access and adhere to HAART. Screening patients served in HIV primarycare clinics for problem drinking is the first step towards this goal. Integrating a substance-abuse screening intervention into routine care visits promotes a culture of comprehensive care, emphasising the importance of attention to this aspect of living with HIV to both patients and providers.

There is currently insufficient evidence to support the fact that adherence is a serious problem in mentally ill patients, and that it justifies mentally ill patients not being initiated on antiretrovirals. Ethically and morally, no mentally ill patient should be denied PICT and the opportunity to initiate antiretroviral treatment regardless of perceived or real barriers to optimal adherence.

\section{Disclosure of positive status to third parties}

It is important to promote openness about HIV, but it is equally important to protect human rights. While it is important that each person has knowledge of their own HIV status, it is also important to assure them that the result of an HIV test is confidential and that decisions about disclosure will be decisions that they must make themselves, rather than somebody else. This is a human rights approach, considering the individual patient's right to privacy, dignity and confidentiality as paramount, and as a right afforded to the mentally ill too.

Therefore, healthcare practitioners should encourage mentally ill HIV-positive patients to disclose their status to their sexual partners. If these patients want to disclose their status, they should also receive counselling first with regard to whom they will tell and when, and to ensure they will be able to cope with the effects of disclosure. ${ }^{[50]}$ If the patient refuses to disclose their status or does not have the capacity to consent, the healthcare practitioner may decide whether to divulge the information to third parties, but only after carefully weighing up all the factors and balancing the patient's human rights against the competing public health goal of preventing possible spread of the disease. Disclosure without the patient's consent must still involve the patient. The practitioner must counsel the patient on the practitioner's ethical obligation to disclose such information. After disclosure, the practitioner must follow up with the patient and the patient's partner to see if disclosure has resulted in adverse consequences or violence for the patient, and, if so, intervene to assist the patient appropriately. ${ }^{[50]}$

\section{Conclusion}

Prior to the existence of antiretroviral treatment, HIV carried a great deal of stigma, and HIV-positive individuals were often victims of discrimination in their homes, their workplaces, and healthcare settings. In order to prevent stigma and discrimination, most HIVtesting programmes around the world developed policies involving informed consent, confidentiality and access to treatment. However, mentally ill patients still continue to be marginalised. Within the context of existing policies and legislations in SA, the authors recommend the promotion of PICT for all psychiatric admissions and mentally ill individuals receiving outpatient services at mental health facilities. This is not only ethically acceptable, but morally imperative. In addition, as long as the risks and benefits are understood, infected mentally ill patients should also be provided with high-quality HIV treatment.

\section{References}

1. UNAIDS. Global Report: UNAIDS Report on the Global AIDS Epidemic 2010. Geneva: UNAIDS, 2010. http://www.unaids.org/documents/20101123.pdf (accessed 10 June 2012).

2. South African Department of Health. National HIV and Syphilis Antenatal Sero-Prevalence Survey in South Africa. Pretoria: DoH, 2010.

3. South African Department of Health. National Prevalence, Incidence, Behaviour and Communication Survey. Pretoria: DoH, 2008.

4. World Health Organization, UNAIDS, UNICEF. Towards Universal Access: Scaling Up Priority HIV/AIDS Interventions in the Health Sector. Progress Report. 2010 Geneva: WHO, 2010. http:// www.who.int/hiv/pub/2010progressreport/en/index.html (accessed 30 June 2012).

5. World Health Organization, UNAIDS, UNICEF. Global HIV/AIDS Response: Epidemic Update and Health Sector Progress Towards Universal Access. Progress Report. Geneva: WHO, 2011. http://www.unaids.org/en/media/unaids/contentassets/documents/ unaidspublication/2011/20111130_ua_report_en.pdf (accessed 22 July 2013).

6. South African National Government. Key Facts of the National Communication Survey on HIV/ AIDS, 2009. Pretoria: Government Printer, 2010. http://www.info.gov.za/issues/hiv/survey_2009. htm\#implications (accessed 30 March 2013).

7. South African Department of Health. National HIV Counselling and Testing (HCT) Policy Guidelines. Pretoria: Government Printer, 2010. http://www.uj.ac.za/EN/CorporateServices/ ioha/Documentation/Documents/hct_policy_guidelines\%202010.pdf (accessed 22 July 2013).

8. World Health Organization, UNAIDS. Guidance on Provider-Initiated HIV Testing and Counselling in Health Facilities. Geneva: WHO, 2007. http://who.int/hiv/pub/ guidelines/9789241595568_en.pdf (accessed 2 April 2013).

9. McQuillan GM, Kruszon-Moran D, Kottiri BJ, et al. Prevalence of HIV in the US household population: The National Health and Nutrition Examination Surveys, 1988 to 2002. J Acquir Immune Defic Syndr 2006;41(5):651-656. [http://dx.doi.org/10.1097/01.qai.0000194235.31078.f6] 


\section{n

10. Henning MP, Krüger C, Fletcher L. HIV sero-positivity in recently admitted and long-term psychiatric in-patients: Prevalence and diagnostic profile. Afr J Psychiatry 2012;15(1):47-53. [http://dx.doi.org/10.4314/ajpsy.v15i1.7]

11. Singh D, Berkman A, Bresnahan M. Seroprevalence and HIV associated factors among adults with severe mental illness - a vulnerable population. S Afr Med J 2009;99(7):523-527.

12. Sebit MB, Tombe M, Siziya S, et al. Prevalence of HIV/AIDS and psychiatric disorders and their related risk factors among adults in Epworth, Zimbabwe. East Afr Med J 2003;80(10):503-512.

13. Meade CS, Sikkema KJ. HIV risk behaviour among adults with severe mental illness: A systematic review. Clin Psychol Rev 2005;25(4):433-457. [http://dx.doi.org/10.1016/Fj.cpr.2005.02.001]

14. Chou R, Huffman LH, Fu R, et al. Screening for HIV: A review of the evidence for the US Preventive Services Task Force. Ann Intern Med 2005;143(1):55-73.

15. Crepaz N, Lyles CM, Wolitski RJ, et al. Do prevention interventions reduce HIV risk behaviours among people living with HIV? A meta-analytic review of controlled trials. AIDS 2006;20(2):143 157. [http://dx.doi.org/10.1097/01.aids.0000196166.48518.a0]

16. Desai MM, Rosenheck RA, Desai RA. Prevalence and correlates of human immunodeficiency virus testing and posttest counseling among outpatients with serious mental illness. J Nerv Ment Dis 2007;195(9):776-780. [http://dx.doi.org/10.1097/NMD.0b013e31814514ad]

17. Satriano J, McKinnon K, Adoff S. HIV service provision for people with severe mental illness in outpatient mental health care settings in New York. J Prev Interv Community 2007;33(1-2):95 108. [http://dx.doi.org/10.1300/J005v33n01_08]

18. Joska JA, Kaliski SZ, Benatar SR. Patients with severe mental illness: A new approach to testing fo HIV. S Afr Med J 2008;98(3):213-217

19. Desai MM, Rosenheck RA. HIV testing and receipt of test results among homeless persons with serious mental illness. Am J Psychiatry 2004;161(12):2287-2294. [http://dx.doi.org/10.1176/appi. ajp.161.12.2287]

20. Goldberg RW. Hepatitis and HIV screening, education, and treatment for adults with serious mental illness. Gen Hosp Psychiatry 2004;26(2):167-168. [http://dx.doi.org/10.1016/j. genhosppsych.2003.08.008]

21. Jürgens R. 'Routinizing' HIV Testing in Low- and Middle-Income Countries - Background Paper New York: Public Health Program of the Open Society Institute, 2006

22. Solomon PL, Tennille JA, Lipsitt D, et al. Rapid assessment of existing HIV prevention programming in a community mental health center. J Prev Interv Community 2007;33(1-2):137 151. [http://dx.doi.org/10.1300/J005v33n01_11]

23. Magongo B, Magwaza S, Mathambo V, et al. National report on the assessment of the public sector's Voluntary Counseling and Testing Programme. Durban: Health Systems Trust, 2002. http://healthlink.org.za/uploads/files/vct_national.pdf (accessed 30 April 2013).

24. World Health Organization, UNAIDS. UNAIDS, WHO Policy Statement on HIV Testing Geneva: WHO, 2004. http://www.who.int/hiv/pub/vct/statement/en/ (accessed 30 April 2013).

25. Senn TE, Carey MP. HIV testing among individuals with a severe mental illness: Review, suggestions for research, and clinical implications. Psychol Med 2009;39(3):355-363. [http:// dx.doi.org/10.1017/S0033291708003930]

26. Van Staden CW, Krüger C. Incapacity to give informed consent owing to mental disorder. J Med Ethics 2003;29(1):41-43

27. Appelbaum PS, Grisso T. MacCAT-CR: MacArthur Competence Assessment Tool for Clinical Research. Sarasota, FL: Professional Resource Press, 2001.

28. Jeste DV, Palmer BW, Appelbaum PS, et al. A new brief instrument for assessing decisional capacity for clinical research. Arch Gen Psychiatry 2007;64(8):966-974. [http://dx.doi. org/10.1001/archpsyc.64.8.966

29. South African National Government. South African National Health Act No. 61 of 2003 Pretoria: Government Printer, 2003. http://www.info.gov.za/view/DownloadFileAction?id=6803 (accessed 30 May 2012).

30. South African National Government. The Mental Health Care Act, No. 17 of 2002. Pretoria Government Printer, 2002. https://www.info.gov.za/view/DownloadFileAction?id=6805 (accessed 30 May 2012)
31. Bogart LM, Kelly JA, Catz SL, Sosman JM. Impact of medical and nonmedical factors on physician decision making for HIV/AIDS antiretroviral treatment. J Acquir Immune Defic Syndr 2000;23:396-404.

32. Uldall KK, Palmer NB, Whetten K, Mellins C, HIV/AIDS Treatment Adherence Health Outcomes and Cost Study Group. Adherence in people living with HIV/AIDS, mental illness, and chemical dependency: A review of the literature. AIDS Care 2004;6:S71-S96. [http://dx.doi.org/10.1080/0 9540120412331315277]

33. Tseng AL, Foisy M. Significant interactions with new antiretrovirals and psychotropic drugs. Ann Pharmacother 1999;33(4):461-473.

34. Himelhoch S, Moore RD, Treisman G, Gebo KA. Does the presence of a current psychiatric disorder in AIDS patients affect the initiation of antiretroviral treatment and duration of therapy? J Acquir Immune Defic Syndr 2004;37(4):1457-1463.

35. Gill CJ, Davidson HH, Simon JL, Thea DM, Sabin LL. No room for complacency about adherence to antiretroviral therapy in sub-Saharan Africa. AIDS 2005;19(12):1243-1249. [http://dx.doi org/10.1097/01.aids.0000180094.04652.3b

36. Boden D, Hurley A, Zhang L, et al. HIV-1 drug resistance in newly infected individuals. JAMA 1999;282(12):1135-1141. [http://dx.doi.org/10.1001/jama.282.12.1135]

37. Wainberg MA, Friedland G. Public health implications of antiretroviral therapy and HIV drug resistance. JAMA 1998. 279: 1977-1983. [http://dx.doi.org/10.1001/jama.279.24.1977]

38. Paterson DL, Potoski B, Capitano B. Measurement of adherence to antiretroviral medications I Acquired Immune Def Syndr 2002;31(3):S103-S106. [http://dx.doi.org/10.1097/00126334200212153-00003]

39. Saferen SA, Kumarasamy N, James R, et al. ART adherence, demographic variables and CD4 outcome among HIV-positive patients on antiretroviral therapy in Chennai, India. AIDS Care 2005;17(7):853-862. [http://dx.doi.org/10.1080/09540120500038439]

40. Osterberg L, Blaschke T. Adherence to medication. N Engl J Med 2005;353(5):487-497. [http:// dx.doi.org/10.1056/NEJMra050100]

41. Nemes MIB, Carvalho HB, Souza MFM. Antiretroviral therapy adherence in Brazil. AIDS 2004;18(3):S15-S20

42. Himelhoch S, Brown $\mathrm{CH}$, Walkup J, et al. HIV patients with psychiatric disorders are less likely to discontinue HAART. AIDS 2009;23(13):1735-1742. [http://dx.doi.org/10.1097/ QAD.0b013e32832b428f]

43. Kumar V, Encinosa W. Effects of antidepressant treatment on antiretroviral regimen adherence among depressed HIV-infected patients. Psychiatr Q 2009;80(3):131-141. [http://dx.doi. org/10.1007/s11126-009-9100-z]

44. Kemppainen JK, Levine R, Buffum M, et al. Antiretroviral adherence in persons with HIV/AIDS and severe mental illness. J Nerv Ment Dis 2004;192(6):395-404.

45. Chander G, Himelhoch S, Moore RD. Substance abuse and psychiatric disorders in HIV-positive patients: Epidemiology and impact on antiretroviral therapy. Drugs 2006;66(6):769-789.

46. Palmer NB, Basinski JR, Uldall KK. Psychiatric illness, access and adherence to HAART: A brief review of recent findings and implications for care. HIV Therapy 2010;4(2):215-230. [http:// dx.doi.org/10.2217/hiv.10.4]

47. Whetten K, Reif S, Ostermann J, et al. Improving health outcomes among individuals with HIV mental illness, and substance use disorders in the Southeast. AIDS Care 2006;18(Suppl. 1):S18S26. [http://dx.doi.org/10.1080/09540120600839330]

48. Battaglioli-DeNero AM. Strategies for improving patient adherence to therapy and long term patient outcomes. J Assoc Nurses AIDS Care 2007;18( Suppl 1):S17-S22. [http://dx.doi. org/10.1016/j.jana.2006.11.020]

49. Moosa MYH, Jonsson G, Jeenah FY, et al. Support groups for HIV-positive mentally il patients. International Journal of Psychology and Counseling 2009;1(9):147-153. http://www. academicjournals.org/ijpc/PDF/Pdf2009/Nov/Moosa\%20et\%20al.pdf (accessed 23 July 2013).

50. Open Society Foundation for South Africa. A Best Practice Guide to HIV Disclosure Pinelands: Open Society Foundation for South Africa, 2009. http://osf.org.za/wp/wp-content/ uploads/2012/09/A-best-pracrtice-guide-to-HIV-disclosurel.pdf. (accessed 2 April 2013). 